\title{
Some problems in the use of the Coulter counter for erythrocyte total counts and volume distribution
}

\author{
H. BRUCE COLLIER \\ From the Division of Clinical Pathology, Department of Pathology, University of Alberta, \\ Edmonton, Canada
}

SYNOPSIS Normal erythrocytes were subject to swelling and some haemolysis when suspended in a $\mathrm{NaCl}$-formaldehyde- $\mathrm{Na}_{2}$ EDTA diluent of $p \mathrm{H} 4.9$ for routine blood-cell counting in the Coulter counter. A phosphate-buffered saline of $p \mathrm{H} 7.4$ was substituted and has proven satisfactory for total erythrocyte counts.

Polyvinyltoluene latex was unsuitable for volume calibration of the counter, because of aggregation of the particles in saline. Ragweed pollen grains gave a calibration factor that agreed well with that for fresh erythrocytes. Recent batches of Dade's Erythro-Trol (a suspension of fixed erythrocytes) were satisfactory for both total counts and volume calibration.

Recently a project was set up for medical undergraduates in which they used the Coulter counter, model $\mathbf{B}^{1}$ to perform total erythrocyte counts and cell-size distributions on their own and on patients' blood. Unexpectedly, the counts were low for normal males, and the mean cell threshold values (MCT, a measure of the mean cell volume, MCV) were about 30, instead of the expected 21 to 22 . Furthermore, it was found that the counts decreased steadily with time, after the 1:50,000 dilutions were made up.

It had also been noted in the routine haematology laboratory of the University of Alberta Hospital that specimens, made up in the routine diluent for some time before counting, occasionally showed significant errors in total erythrocyte count. Quality control at that time had involved checking the hospital's Coulter counter against haemacytometer counts each day; but as the dilutions of control blood were usually counted first, in advance of the specimens from patients, any deleterious effect of the diluent usually remained undetected.

The routine hospital diluent, which had also been used by the medical students in their project, was found to have a $p \mathrm{H}$ of 4.9. Hampson and Maizels (1926) demonstrated that erythrocytes in phosphate buffers had a minimum volume at $p \mathbf{H} 8 \cdot 1$, which

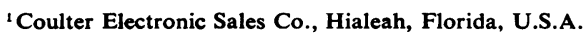

Received for publication 16 May 1967. increased to a maximum at $p H$ 5.4. Van Kampen, Graafland, and Hasselman (1957) studied the $p H$ resistance of erythrocytes and found that the average $p \mathrm{H}$ of beginning haemolysis was 5.15 in normal specimens, but approached neutrality in certain anaemias. These findings explain the low counts and cell swelling that we encountered with the routine diluent of $p \mathrm{H} \mathrm{4.9.}$

\section{MATERIALS}

1 Erythro-Trol, from Dade Reagents Inc., Miami, Fla., U.S.A.

2 Celltrol, from Pfizer Diagnostics Dept., Chas. Pfizer and Co., New York, N.Y., U.S.A.

3 Polyvinyltoluene Latex, 3.49 $\mu$ mean diameter, from the Bioproducts Dept., Dow Chemical Co., Midland, Mich., U.S.A.

4 Ragweed pollen, from the Hollistier-Stier Laboratories, Dallas, Texas, U.S.A.

MODified EAGLE'S MEDIUM $\mathrm{NaCl}, 6.2 \mathrm{~g}, 0.36 \mathrm{~g}$ of $\mathrm{KCl}$, $0.13 \mathrm{~g}$ of $\mathrm{NaH}_{2} \mathrm{PO}_{4} \cdot \mathrm{H}_{2} \mathrm{O}, 2.0 \mathrm{~g}$ of $\mathrm{NaHCO}_{3}, 0.18 \mathrm{~g}$ of $\mathrm{CaCl}_{2}, 0.15 \mathrm{~g}$ of $\mathrm{MgCl}_{2} \cdot 6 \mathrm{H}_{2} \mathrm{O}$, and $0.9 \mathrm{~g}$ of dextrose were dissolved in distilled water and made up to 1 litre. The solution was $p \mathrm{H} 8 \cdot 1,287$ milliosmoles/litre. It was filtered through a $0 \cdot 8 \mu$ Millipore filter before use.

ISOTON A diluent from Coulter Electronic Sales Co., Hialeah, Fla., U.S.A.

ACID DILUENT $\mathrm{NaCl}, 9 \mathrm{~g}, 75 \mathrm{mg}$ of disodium EDTA, and $2.5 \mathrm{ml}$ of $37 \%$ formaldehyde were dissolved in distilled water and made up to 1 litre; $p H 4.9$. 
NeUtral dilueNT $\mathrm{NaCl}, 7 \cdot 7 \mathrm{~g}, 0.55 \mathrm{~g}$ of $\mathrm{NaH}_{2} \mathrm{PO}_{4} \cdot \mathrm{H}_{2} \mathrm{O}$, $2.27 \mathrm{~g}$ of $\mathrm{Na}_{2} \mathrm{HPO}_{4}$, and $2.5 \mathrm{ml}$ of $37 \%$ formaldehyde were made up in distilled water and diluted to 1 litre; pH 7·4.

\section{METHODS}

Since threshold units, $T$, on the threshold dials of the counter are directly proportional to particle volume, the dials can be calibrated to read in volume units, $\mu^{3}$, through multiplying $T$ by a calibration factor. Our counter was calibrated by the method of Brecher, Jacobek, Schneiderman, Williams, and Schmidt (1962), also outlined in the review by Pruden and Winstead (1964).

The mean erythrocyte volume (MCV) of fresh normal human blood was obtained from the erythrocyte count (in the Coulter counter) and the haematocrit reading (heparinized blood centrifuged in blood-collecting tubes of $1.5 \mathrm{~mm}$ bore and $150 \mathrm{~mm}$ length in a MSE clinical centrifuge at full speed for $\mathbf{4 5}$ minutes, relative centrifugal force about $1500 \times g$; the readings agreed within $1 \%$ with those obtained in a microhaematocrit centrifuge run for 10 minutes at about $5000 \times g$ ).

Of this blood, a $1: 50,000$ dilution was made in modified Eagle's medium which, according to Brecher et al. (1962), maintains the same erythrocyte sizes as in plasma. From the cell-size distribution curve, the mean cell threshold, MCT (we prefer this abbreviation to $\overline{\mathbf{W}}$ ), was computed; this, divided into the MCV, gives the calibration factor, $a$. Thus, for any threshold reading, $T$, the corresponding volume (in $\mu^{3}$ ) is $\mathbf{T} \times a$. The MCT values could be replicated to within $1 \%$ on the same cell suspension. The instrument settings for the erythrocyte calibrations are given in Table I.

One problem was encountered that we have not seen mentioned elsewhere. The cell counts for a given window size, W, were not equal, as they should have been, when the separate-locked switch ' $C$ ' was in the separate and

\section{TABLE I}

SUMMARY OF INSTRUMENT SETTINGS, MEAN THRESHOLDS, AND CALIBRATION CONSTANTS FOR VARIOUS TYPES OF PARTICLES ${ }^{1}$

\begin{tabular}{|c|c|c|c|c|c|}
\hline Particle & $\begin{array}{l}\text { Mean } \\
\text { Volume } \\
\left(\mu^{3}\right)\end{array}$ & $I / A M P$ & $M C T$ & $a$ & $a \times A M P$ \\
\hline $\begin{array}{l}\text { Latex } \\
\text { Ragweed pollen } \\
\text { Ragweed pollen } \\
\text { Erythrocytes }\end{array}$ & $\begin{array}{r}22 \cdot 3 \\
3260 \\
3880 \\
88\end{array}$ & $\begin{array}{r}1 / 1 \\
16 / 1 \\
16 / 1 \\
1 / 1\end{array}$ & $\begin{array}{l}8 \cdot 5 \cdot 9 \cdot 0 \\
43 \cdot 1 \\
53 \cdot 4 \\
19 \cdot 5\end{array}$ & $\begin{array}{l}2 \cdot 5-2 \cdot 6 \\
75 \cdot 6 \\
72 \cdot 7 \\
4 \cdot 52\end{array}$ & $\begin{array}{l}2 \cdot 5-2 \cdot 6 \\
4 \cdot 7 \\
4 \cdot 5 \\
4 \cdot 5\end{array}$ \\
\hline $\begin{array}{l}\text { Erythro-Trol-12 } \\
\text { Erythro-Trol-13 } \\
\text { Erythro-Trol-14 } \\
\text { Erythrocytes } \\
\text { Erythrocytes }\end{array}$ & $\begin{array}{l}52^{2} \\
52^{2} \\
70^{2} \\
92 \\
93\end{array}$ & $\begin{array}{l}1 / 2 \\
1 / 2 \\
1 / 2 \\
1 / 1 \\
1 / 1\end{array}$ & $\begin{array}{l}31 \cdot 7 \\
33 \cdot 7 \\
33 \cdot 8 \\
23 \cdot 0 \\
22 \cdot 8\end{array}$ & $\begin{array}{l}1 \cdot 68 \\
1 \cdot 55 \\
2 \cdot 07 \\
4 \cdot 04 \\
4 \cdot 08\end{array}$ & $\begin{array}{l}3 \cdot 4 \\
3 \cdot 1 \\
4 \cdot 1 \\
4 \cdot 0 \\
4 \cdot 1\end{array}$ \\
\hline
\end{tabular}

${ }^{2}$ Coulter Counter, Model B. $100 \mu$ aperture; $1 / \mathrm{APC}=0 \cdot 707$, gain control at 60 .

2 Manufacturer's stated values for Erythro-Trol.

Abbreviations: APC $=$ aperture current; $\mathbf{A M P}=$ amplification; MCT $=$ mean cell threshold; $a$ =calibration constant. The values in the lower portion of the Table were obtained after recalibration of the instrument. in the locked position. For example, at a window of 10 in the separate position (lower and upper dial readings of 15 and 25, in T units), a count was 21,399; in the locked position (dial settings of 15 and 100), the count was 21,861. The discrepancy became greater as the window size was decreased: for a window of $2 \cdot 0$, the count in the separate position (settings of 18 and 20 ) was 4,455 ; in the locked position (settings also 18 and 20) it was 5,493.

This discrepancy was corrected by the Coulter representative who adjusted the 25 kilo-ohm potentiometer which is wired to the separate-locked switch. (This is normally a factory adjustment; see the wiring diagram. ${ }^{2}$ ) The dials A and B were set at 18 and 20, which gives a window of $2 \cdot 0$, whether the switch is in the separate or locked position. The potentiometer was then adjusted until the counts were equal in the two switch positions. A model B counter in another laboratory gave, without adjustment, counts that agreed with $1 \%$ in the two switch positions.

\section{RESULTS}

TOTAL ERYTHROCYTE COUNTS Since the routine diluent of $p \mathrm{H} \mathrm{4.9}$ had caused swelling and haemolysis the hospital laboratory adopted the diluent buffered with $\mathrm{M} / 50$ phosphate, $p \mathrm{H} 7 \cdot 4$. This was used unmodified for routine total erythrocyte counts. For leucocyte counts, $\mathrm{Na}_{2}$ EDTA was added with the saponin, since Magath and Berkson (1960) stated that " . . . saponin would not lake the blood properly except when EDTA was used'.

Results with the old diluent of $p H 4.9$ and the new diluent of $p \mathrm{H} \mathrm{7.4}$ were then compared with those from the modified Eagle's medium as a standard. (Modified Eagle's medium is claimed to give normal erythrocyte volumes.) Total counts and half-counts, $\mathbf{T}_{50}$, were compared. The half-count (Pruden and Winstead, 1964) is the threshold setting at which the count is exactly $50 \%$ of the total. It is roughly proportional to the MCV and can be used for rapid measurements of volume changes.

Typical results on 1:50,000 blood dilutions allowed to stand one hour before counting were as follows, compared with the initial counts. The total count fell by $1.4 \%$ in modified Eagle's medium, by $1.1 \%$ in the $p \mathrm{H} 7.4$ diluent, and by $11 \%$ in the $p \mathrm{H} 4.9$ diluent. $T_{50}$ values increased by $3 \%$ in the neutral diluent and by $33 \%$ in the acid medium, as compared with modified Eagle's medium.

Various lots of Erythro-Trol (a suspension of fixed erythrocytes) gave counts that agreed with the rated values within $1 \%$. The haematology laboratory of the University of Alberta Hospital attempted to use a 1:50,000 suspension of Erythro-Trol in routine diluent for quality control of erythrocyte counts

I have since drilled a $\frac{1}{4}$-inch hole in the left side of the instrument case, in order to be able to insert a screwdriver blade for adjusting this potentiometer. 
throughout the day. It was found, however, by Dr. J. R. Hill and Dr. Ishbel Leduc, that it was impossible to maintain a uniform suspension for more than three hours, in spite of repeated shaking or continuous stirring; irreversible aggregation seemed to take place. Dutra (1966) has recently described a similar experience. Since that time we were provided with a batch of Celltrol by the manufacturer, and it does provide a more stable $1: 50,000$ suspension.

The haematology laboratory now uses whole blood (with EDTA as anticoagulant), kept in the refrigerator, for day-to-day quality control; the total erythrocyte count remains constant for at least one week.

volume CALIBRATION As mentioned under Methods, the counter was calibrated for cell-volume readings by using fresh blood of known MCV, suspended in modified Eagle's medium. However, an independent volume calibration was desired, and several types of particles of known size were used. The results are summarized in Table $\mathrm{I}$.

Latex The polyvinyltoluene latex was of $3.49 \mu$ mean particle diameter. Aggregation of the particles in the saline medium could not be prevented, however, even by addition of a surfactant provided by the Dow Company, and further attempts at its use were abandoned.

Ragweed pollen The pollen grains were stated to be $19-20 \mu$ in diameter. They were soaked in ethanol and then suspended in modified Eagle's medium, but the mean diameter, as measured with an ocular micrometer, varied from batch to batch. Two batches gave mean diameters of $18.4 \pm 0.8(\mathrm{SD})$ and $19.5 \pm$ $0.8 \mu$, corresponding to mean volumes of 3260 and $3880 \mu^{3}$, respectively.

Volume distribution curves were obtained at an amplification setting of $1 /$ AMP $=16$. Mean cell threshold values of 43.1 and 53.4 were obtained for the two batches of pollen, giving $a$ values of 75.7 and 72.6. These, when divided by 16 to correspond to the amplification of $1 / 1$ used for erythrocytes, give 4.7 and $4 \cdot 5$, values that are acceptably close to the $a$ values of 4.4-4.5 obtained with erythrocytes at that time.

Erythro-Trol Late in 1965 a supply of ErythroTrol was received from the Coulter Electronic Sales Co. of Canada, Toronto. This gave a total count of 4.70 million per $\mu l$. (rated count 4.75 ). Size distribution curves were obtained at $1 / \mathrm{AMP}=\frac{1}{2}$. The MCT was 31.7 and the stated MCV was $52 \mu^{3}$, which gives $a=1.65$, or 3.3 for the usual erythrocyte setting. This was lower than expected and recalibration with fresh blood at the same settings gave $a=$ $2 \cdot 02,2 \cdot 04$.

Correspondence with Dade Reagents, Inc., re- vealed that the MCV values on early lots of ErythroTrol (up to lot no. 13) were erroneously low, owing to their use of unbuffered saline in preparing calibration curves with fresh blood. The calibrations are now said to be done in modified Eagle's medium.

A new lot, ERT-14, gave a total count of 4.70 (rated at 4.72). The MCT from our distribution curve was 34.0 , which at the stated MCV of $70 \mu^{3}$ gives $a=2.07$. This is equivalent to 4.14 at our erythrocyte setting, which agreed well with our calibration factor at that time of $4 \cdot 0-4 \cdot 1$. (See Table I for a summary of calibration factors for different types of particles. In the last column of the Table the calculated $a$ values have been multiplied by the amplification, to make them directly comparable with the values for erythrocytes, at $1 / \mathrm{AMP}=1 / 1$.)

Recently the Coulter Company sent us a supply of their new diluent, Isoton, to be used instead of modified Eagle's medium. We found it to be $p \mathrm{H} \mathrm{8.2,}$ 280 milliosmoles per litre. When fresh blood was diluted in Isoton and in modified Eagle's medium the total erythrocyte counts were 4.70 and 4.69 , respectively. The MCT was $20 \cdot 8$ in Isoton and $21 \cdot 1$ in modified Eagle's medium, a difference of only $1.4 \%$. After standing for one-and-a-half hours at the 1:50,000 dilution, the counts were 4.59 in Isoton and 4.49 in modified Eagle's medium.

\section{DISCUSSION}

In our experience, the most important consideration in obtaining reliable erythrocyte counts and volume distributions is the $p \mathrm{H}$ of the diluent. It must not fall below 7 , and should be about 8 . Publications on the use of the Coulter counter have largely ignored the effects of $p \mathrm{H}$. The most comprehensive review on the counter is that of Pruden and Winstead (1964). They mentioned that addition of formaldehyde to a saline medium may cause swelling and lysis, possibly owing to the presence of formic acid; but they did not give the $p \mathrm{H}$ of their diluent or propose neutralizing it. They did recommend the addition of $0.044 \%$ albumin to the formol-saline, as suggested by Brecher, Schneiderman, and Williams (1956) (who actually added $0.005 \%$ ). Peacock, Williams, and Mengoli (1960) used a modified Krebs-Ringer medium buffered with $0.01 \mathrm{M}$ phosphate and containing $0.05 \%$ serum albumin; the $p H$ was not stated, but it would probably be about 7.4-7.5.

Brecher et al.(1956) mentioned that 1: 50,000 dilutions are somewhat unstable, even in modified Eagle's medium. Thus, in routine cell counting it is highly undesirable to prepare a number of such dilutions and allow them to stand until the counting is finally completed. If a two-step dilution procedure is used, the 1:500 dilutions may be allowed to stand, 
as they are more stable. The final dilution should be done just before the count.

For volume calibration of the counter, fresh blood in modified Eagle's medium or Isoton may be used if the MCV is accurately known. While early lots of Dade Erythro-Trol were incorrectly calibrated for volume, the product now seems to be satisfactory.

It must be strongly emphasized that a suspension of fixed erythrocytes, such as Erythro-Trol or Celltrol, may be satisfactory for checking dilutions and the behaviour of the counter but that it cannot replace fresh blood in revealing a fault in the diluent, such as incorrect $p \mathrm{H}$ or osmolality.

This work was supported by a grant from the Medical Research Council of Canada. Many helpful discussions were held, on these problems, with Dr. R. E. Bell, Director of the Department of Clinical Laboratory Services, University of Alberta Hospital, and with Mr. A. Eaton, former Haematology Supervisor. The Coulter Company provided much appreciated assistance. Polyvinyltoluene latex samples were kindly supplied by $\mathbf{M r}$. L. J. Lippie of the Dow Chemical Company. Dr. A. G. Stewart, of the Department of Paediatrics, kindly permitted us to compare counts on his Coulter Counter, Model B, with our instrument. He was also good enough to review the manuscript of this paper. The skilled technical assistance of Mr. G. Duchon is gratefully acknowledged.

\section{ADDENDUM}

I have recently acquired a $50 \mu$ aperture tube and a Coulter MCV computer for the study of cell-size

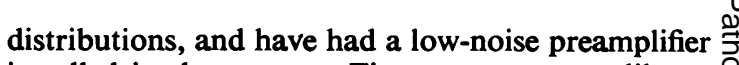
installed in the counter. First attempts to calibrate the MCV computer with erythrocytes gave very dis- $\overrightarrow{\vec{A}}$ cordant results. Instrument settings were: preamplifier control 32-H, 1/AMP 4, 1/APC $\frac{1}{2}(=1$ milliampere).

It was finally discovered that this aperture current

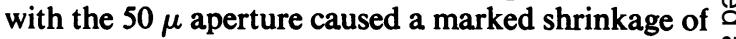
the erythrocytes, although the total count was not is affected. Erythro-Trol was not altered in volume. As $\overrightarrow{0}$ compared with the $100 \mu$ aperture, the current density is increased fourfold, and the time interval in passing through the aperture is also increased fourfold.

Cells that had passed through the aperture were collected, and the suspension was passed through is the instrument a second time. The readings indicated $\vec{V}$ that the current of 1 ma. had caused almost complete disintegration of the erythrocytes.

Instrument settings of $1 / \mathrm{AMP}=1,1 / \mathrm{APC}=2$ are now giving satisfactory results.

\section{REFERENCES}

Brecher, G., Jacobek, E. F., Schneiderman, M. A., Williams, G. Z., and Schmidt, P. J. (1962). Ann. N. Y. Acad. Sci., 99, 242. —, Schneiderman, M., and Williams, G. Z. (1956). Amer. J. clin. Path., 26, 1439.

Dutra, F. R. (1966). Ibid., 46, 286.

Hampson, A. C., and Maizels, M. (1926). J. Physiol. (Lond.), 62, xvi. Magath, T. B., and Berkson, J. (1960). Amer. J. clin. Path., 34, 203.

Peacock, A. C., Williams, G. Z., and Mengoli, H. F. (1960). J. nat. Cancer Inst., 25, 63.

Pruden, E. L., and Winstead, M. E. (1964). Amer. J. med. Technol., 30, 1.

Van Kampen, E. J., Graafland, C. A., and Hasselman, J. J. F. (1957). Clin. chim. Acta, 2, 95. 